\title{
Recent results from PHENIX on jet suppression and direct photon production
}

\author{
Victor Riabov (for the PHENIX collaboration) \\ PNPI \\ Orlova roscha 1, Gatchina, Russia \\ E-mail: riabovvgepnpi.spb.ru
}

It has been established that a new state of matter called strongly interacting quark-gluon plasma (sQGP) is created in heavy ion collisions at RHIC energies. Detailed study of the properties of this hot and dense matter has been a primary purpose of research at RHIC over the last years. In this proceeding we present recent PHENIX results for system size and energy dependence of high $\mathrm{p}_{\mathrm{T}}$ hadron suppression in heavy ion collisions. Dependence of intermediate $\mathrm{p}_{\mathrm{T}}$ hadron production on particle mass and quark content is discussed. We also report latest results for direct photon production along with centrality dependence of soft direct photon yields, elliptic and triangular flow at low momentum.

XXII International Baldin Seminar on High Energy Physics Problems

15-20 September 2014

JINR, Dubna, Russia

(C) Copyright owned by the author(s) under the terms of the Creative Commons Attribution-NonCommercial-ShareAlike Licence. http://pos.sissa. it/ 


\section{Introduction}

Hard scattered partons loose significant part of their energy traversing the medium produced in heavy ion collisions resulting in suppressed production of high $\mathrm{p}_{\mathrm{T}}$ hadrons or jet quenching. As a result high $\mathrm{p}_{\mathrm{T}}$ hadrons and jets are a sensitive probe of opacity of the produced medium. PHENIX has measured production of high $\mathrm{p}_{\mathrm{T}}$ identified hadrons $\left(\pi^{0}, \eta, \mathrm{K}^{*}, \mathrm{~K}_{\mathrm{s}}, \omega, \phi\right)$ in $\mathrm{d}+\mathrm{Au}, \mathrm{Cu}+\mathrm{Cu}$ and $\mathrm{Au}+\mathrm{Au}$ collisions at different energies. Obtained results are used to estimate parton energy loss in the hot and dense medium, its dependence on collision centrality and interaction energy.

At intermediate $\mathrm{p}_{\mathrm{T}}$ hadron production is driven by competing particle production mechanisms which are sensitive as to high $\mathrm{p}_{\mathrm{T}}$ phenomena as to collective effects in the produced medium which are a subject of special interest. Detailed study of hadron suppression for mesons and baryons with different masses and quark content in this kinematic region helps to determine the leading particle production mechanisms.

The electromagnetic probes such as direct photons are not affected by strong nuclear forces. They are extremely valuable in study of the jet quenching phenomena. In addition measurement of direct photon produced at all stages of the interaction allows to constrain the time evolution of the medium.

\section{Production of hadrons at high transverse momentum}

Invariant differential production spectra were measured for identified hadrons in $\mathrm{p}+\mathrm{p}$ and heavy ion collisions. Potential collective effects in nuclear collisions are numerically studied with nuclear modification factor $\mathrm{R}_{\mathrm{AA}}$, which is a ratio of the yields measured for the same particle in heavy ion and $p+p$ collisions and scaled by the corresponding number of inelastic nucleon-nucleon collisions $\mathrm{N}_{\text {coll }}$. Number of such collisions is not experimentally measured and is estimated using Glauber model [1].

In Fig.1 you can see nuclear modification factors, $R_{\mathrm{dA}}$, measured for $\pi^{0}, \mathrm{~K}_{\mathrm{s}}, \mathrm{K}^{*}, \phi$ mesons and protons in $\mathrm{d}+\mathrm{Au}$ collisions at ${\sqrt{\mathrm{S}_{\mathrm{NN}}}}=200 \mathrm{GeV}$ as a function of transverse momentum [2]. The top panel of the figure corresponds to most central collisions and panel on the bottom is for most peripheral ones. In peripheral collisions nuclear modification factors are consistent with unity for all hadrons at $\mathrm{p}_{\mathrm{T}}>2 \mathrm{GeV} / \mathrm{c}$ and all particles are consistent with each other within uncertainties. At the same time in most central collisions the $\mathrm{R}_{\mathrm{dA}}$ for all mesons is the same with a hint of modest Cronin enhancement at intermediate momentum, $2<\mathrm{p}_{\mathrm{T}}<5 \mathrm{GeV} / \mathrm{c}$, and suppression at $\mathrm{p}_{\mathrm{T}}>6 \mathrm{GeV} / \mathrm{c}$. Production of baryons, in this case protons, is strongly enhanced by about a factor of two. The Cronin enhancement is weaker than that previously observed at SPS. However as in the case of lower collision energies enhancement of protons is much stronger comparing to mesons. This observation is hard to reconcile with assumption that Cronin effects originates from soft multiple rescattering of partons in the initial state. A series of models appeared on the market that try to explain Cronin effect by quark recombination in the final state [3].

Recently PHENIX obtained new measurements of the nuclear modification factors in $\mathrm{Au}+\mathrm{Au}$ collisions at ${\sqrt{\mathrm{S}_{\mathrm{NN}}}}=200 \mathrm{GeV}[4,5]$. In Fig.2 you can see comparison of the $\mathrm{R}_{\mathrm{AA}}$ factors measured for neutral pions as a function of transverse momentum at different centralities. Black 
and red points correspond to different data sets. Results are in very good agreement and fully confirm previous observations. These figures illustrate system size dependence of hadron suppression. In peripheral collisions production of neutral pions is slightly suppressed, the $\mathrm{R}_{\mathrm{AA}}$ factor is equal to $\sim 0.8$ with practically no dependence on transverse momentum. Suppression increases with centrality. In most central collisions suppression is the largest at $6-8 \mathrm{GeV} / \mathrm{c}$ $\left(\mathrm{R}_{\mathrm{AA}} \sim 0.2\right)$ and then decreases at higher and lower momenta. Similar behavior was also observed at much higher collision energies at the LHC.

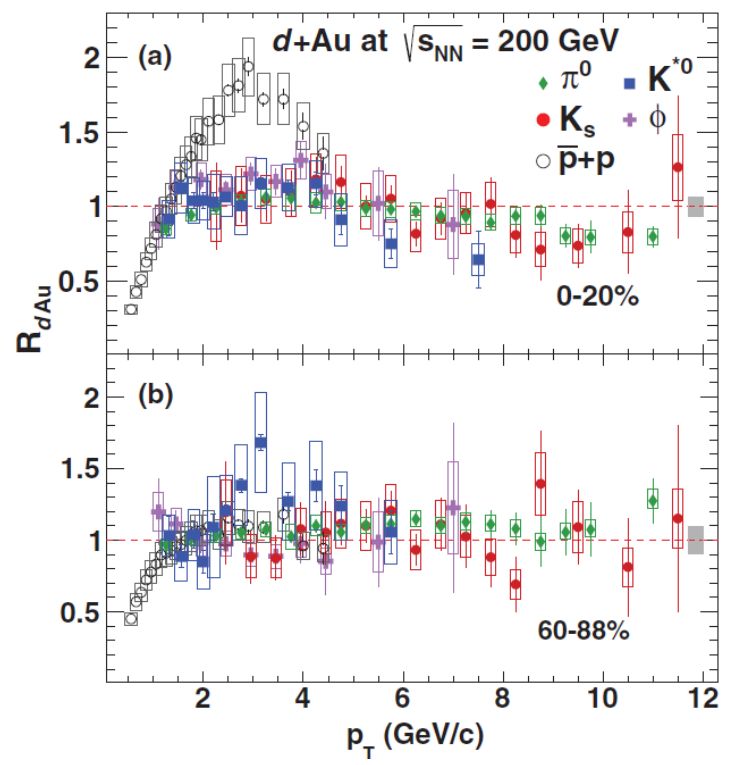

Fig. 1. The nuclear modification factors $\mathrm{R}_{\mathrm{dA}}$ for different hadrons measured in central (a) and peripheral (b) $d+A u$ collisions at $\sqrt{ }_{\mathrm{NNN}_{\mathrm{N}}}=200 \mathrm{GeV}$.

Measurements for neutral pions proved to be very important and provide the widest $\mathrm{p}_{\mathrm{T}}$ coverage. However it is also important to look at species dependence of high $\mathrm{p}_{\mathrm{T}}$ hadron suppression as from physical point of view (for example to see how jet quenching works for different flavors) as from point of view of systematic uncertainties which are different for particles measured using different detector subsystems. In Fig. 3 you can see nuclear modification factors measured for different hadrons at high transverse momentum in $\mathrm{Cu}+\mathrm{Cu}$ and $\mathrm{Au}+\mathrm{Au}$ collisions at ${\sqrt{\mathrm{S}_{\mathrm{NN}}}}=200 \mathrm{GeV}$. Plot on the left shows $\mathrm{R}_{\mathrm{AA}}$ factors for $\pi^{0}, \mathrm{~K}^{*}, \mathrm{~K}_{\mathrm{s}}$ and $\phi$ mesons in $\mathrm{Cu}+\mathrm{Cu}$ collisions. At high $\mathrm{p}_{\mathrm{T}}$ we observe the same level of suppression for all hadrons. Plot on the right shows dependence of the $\mathrm{R}_{\mathrm{AA}}$ integrated at $\mathrm{p}_{\mathrm{T}}>7 \mathrm{GeV} / \mathrm{c}$ on collision centrality for $\pi^{0}, \eta$ and $\omega$ mesons in $\mathrm{Cu}+\mathrm{Cu}$ and $\mathrm{Au}+\mathrm{Au}$ collisions. First we see that dependence of suppression on collision centrality is the same for all these particles. Second observation is that results for $\mathrm{Cu}+\mathrm{Cu}$ and $\mathrm{Au}+\mathrm{Au}$ collisions follow the same universal curve. These results show that suppression of light hadrons at high $\mathrm{p}_{\mathrm{T}}$ does not depend on hadron mass or quark content.

Another interesting question is dependence of jet quenching on collision energy. Some results of the Beam Energy Scan at RHIC are presented in Fig. 4. Plot on the left shows nuclear modification factors measured for neutral pions in most central $\mathrm{Cu}+\mathrm{Cu}$ collisions at $\sqrt{ }_{\mathrm{S}_{\mathrm{NN}}}=22,62$ and $200 \mathrm{GeV}$ [6]. At 200 and $62 \mathrm{GeV}$ production of neutral pions is suppressed 
and at $22 \mathrm{GeV}$ we observe a significant enhancement instead. Plot on the right shows $\mathrm{R}_{\mathrm{AA}}$ factors measured in central $\mathrm{Au}+\mathrm{Au}$ collisions at ${\sqrt{\mathrm{S}_{\mathrm{NN}}}}=39,62$ and $200 \mathrm{GeV}$ with black, blue and red markers [7]. We can see that at all collision energies production of pions is significantly suppressed. At this suppression at 39 and $62 \mathrm{GeV}$ is the same at high transverse momentum. So in heavy ion collisions enhancement takes over suppression somewhere in a range of collision energies between 22 and $39 \mathrm{GeV}$. It is interesting to note that the lowest energy of $22 \mathrm{GeV}$ is very close to the top SPS energy where suppression was not observed also. Comparison of nuclear modification factors measured for charged hadrons at $\mathrm{LHC}$ in $\mathrm{Pb}+\mathrm{Pb}$ collisions at ${\sqrt{\mathrm{S}_{\mathrm{NN}}}}=2.76 \mathrm{TeV}[8]$ and for neutral pions in $\mathrm{Au}+\mathrm{Au}$ collisions at ${\sqrt{\mathrm{S}_{\mathrm{NN}}}}=200 \mathrm{GeV}$ shows that regardless of the difference by a factor of 14 in collision energies the measured factors are very close and they show very similar dependence on transverse momentum in peripheral and central collisions.

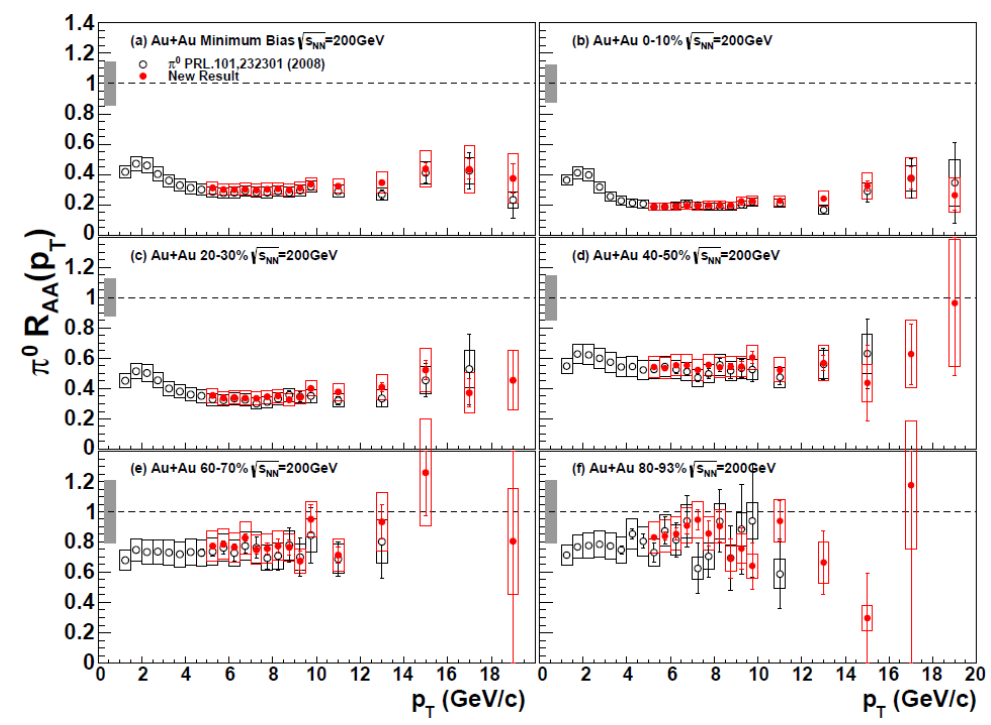

Fig. 2. The nuclear modification factors $R_{A A}$ for neutral pions in $A u+A u$ collisions at ${\sqrt{\mathrm{S}_{\mathrm{NN}}}}=200 \mathrm{GeV}$. Results are presented for different centrality bins and analyses.
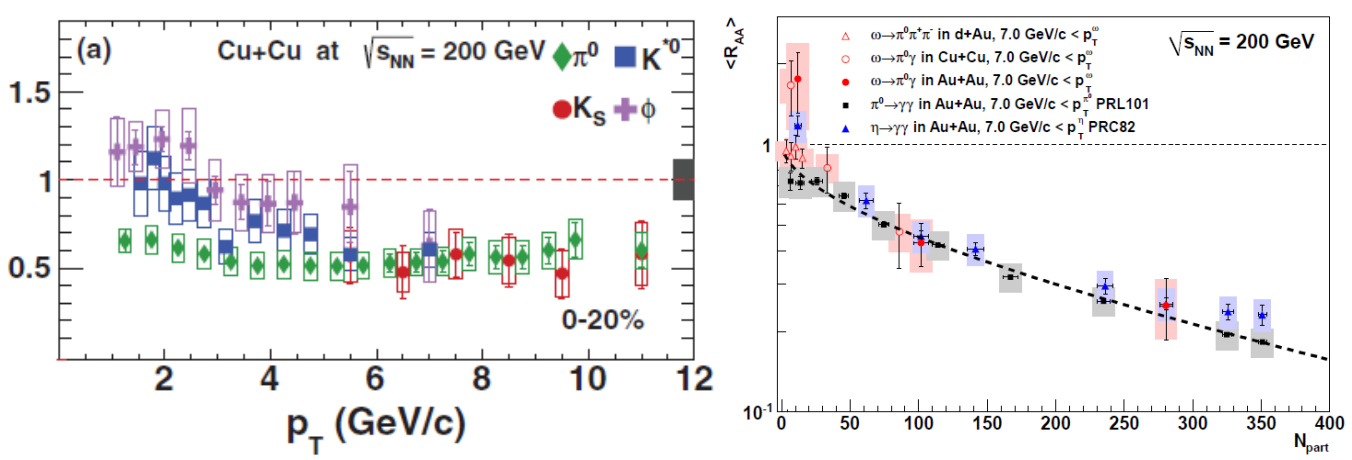

Fig.3. Left: The nuclear modification factors $\mathrm{R}_{\mathrm{AA}}$ for $\pi^{0}, \mathrm{~K}^{*}, \mathrm{~K}_{\mathrm{s}}$ and $\phi$ mesons in central $\mathrm{Cu}+\mathrm{Cu}$ collisions at ${\sqrt{\mathrm{S}_{\mathrm{NN}}}}=200 \mathrm{GeV}$. Right: Dependence of the $\mathrm{R}_{\mathrm{AA}}$ integrated at $\mathrm{p}_{\mathrm{T}}>7 \mathrm{GeV} / \mathrm{c}$ on centrality for $\pi^{0}, \eta$ and $\omega$ mesons in $\mathrm{Cu}+\mathrm{Cu}$ and $\mathrm{Au}+\mathrm{Au}$ collisions at ${\sqrt{\mathrm{S}_{\mathrm{NN}}}}=200 \mathrm{GeV}$. 

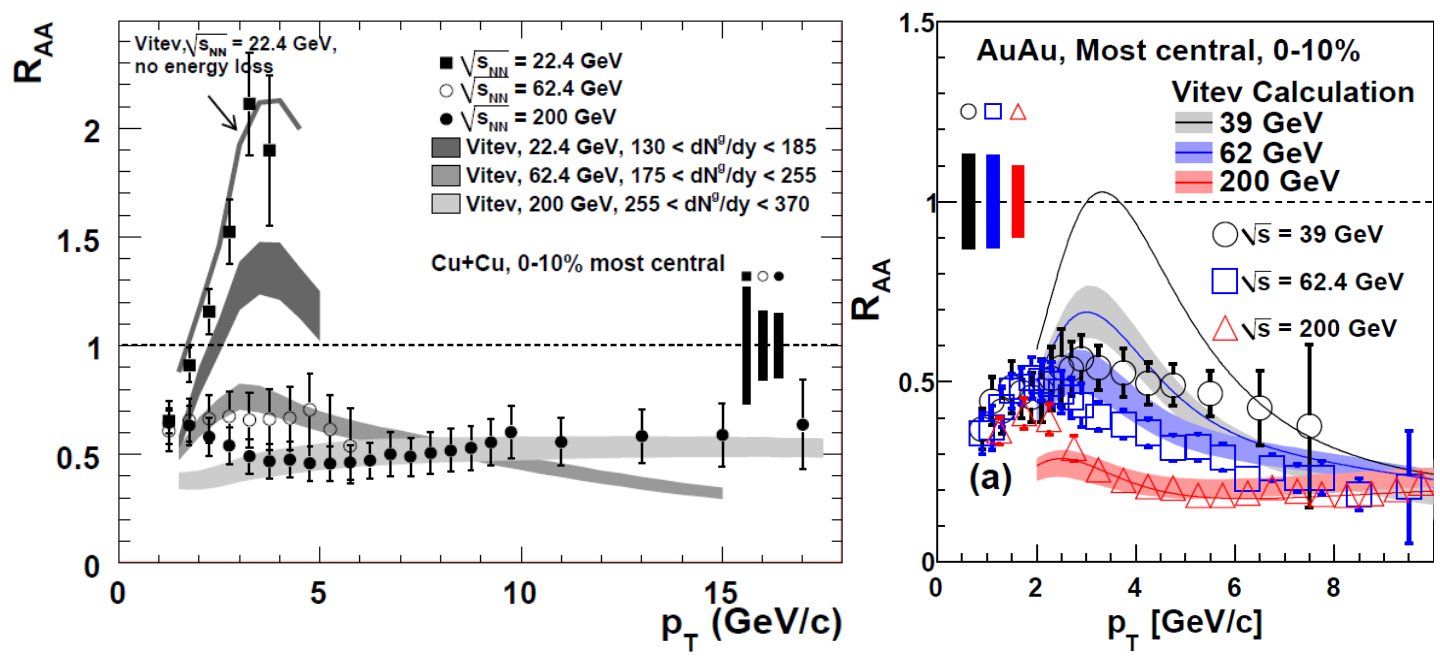

Fig.4. The nuclear modification factors measured for neutral pions in $\mathrm{Cu}+\mathrm{Cu}$ and $\mathrm{Au}+\mathrm{Au}$ collisions at different energies as a function of transverse momentum.

Similarity of the nuclear modification factors measured in heavy ion collisions at different energies indicates that this parameter is biased by the shapes of particle production spectra at different energies. Instead it was suggested to look at fractional momentum loss [9]. Fig. 5 shows dependence of fractional momentum loss on charged particle multiplicity as a measure of energy density. Points of different color correspond to $\mathrm{Cu}+\mathrm{Cu}$ collisions at ${\sqrt{\mathrm{N}_{\mathrm{NN}}}}=200 \mathrm{GeV}$ (black points), $\mathrm{Au}+\mathrm{Au}$ collisions at ${\sqrt{\mathrm{S}_{\mathrm{NN}}}}=62$ and $200 \mathrm{GeV}$ (magenta and red points) and $\mathrm{Pb}+\mathrm{Pb}$ collisions at $\sqrt{ }_{\mathrm{S}_{\mathrm{NN}}}=2.76 \mathrm{TeV}$ (blue points). We can see that regardless of the similarity in $\mathrm{R}_{\mathrm{AA}}$ values the fractional momentum losses are quite different for all systems. Momentum losses increase by $50 \%$ from central $\mathrm{Au}+\mathrm{Au}$ collisions at ${\sqrt{\mathrm{S}_{\mathrm{NN}}}}=200 \mathrm{GeV}$ to most central $\mathrm{Pb}+\mathrm{Pb}$ collisions at ${\sqrt{\mathrm{S}_{\mathrm{NN}}}}=2.76 \mathrm{TeV}$ and by a factor of 6 from ${\sqrt{\mathrm{S}_{\mathrm{NN}}}}=62 \mathrm{GeV}$ to ${\sqrt{\mathrm{S}_{\mathrm{NN}}}}=2.76 \mathrm{TeV}$. Momentum losses gradually increase with multiplicity and all measured points independent of collision energy merge to one universal curve at large values of particle multiplicity. We can also see that at the same energy points corresponding to different colliding nuclei follow the same scaling, like black and red points obtained for $\mathrm{Au}+\mathrm{Au}$ and $\mathrm{Cu}+\mathrm{Cu}$ collisions at $\sqrt{ }_{\mathrm{NN}}=200 \mathrm{GeV}$.

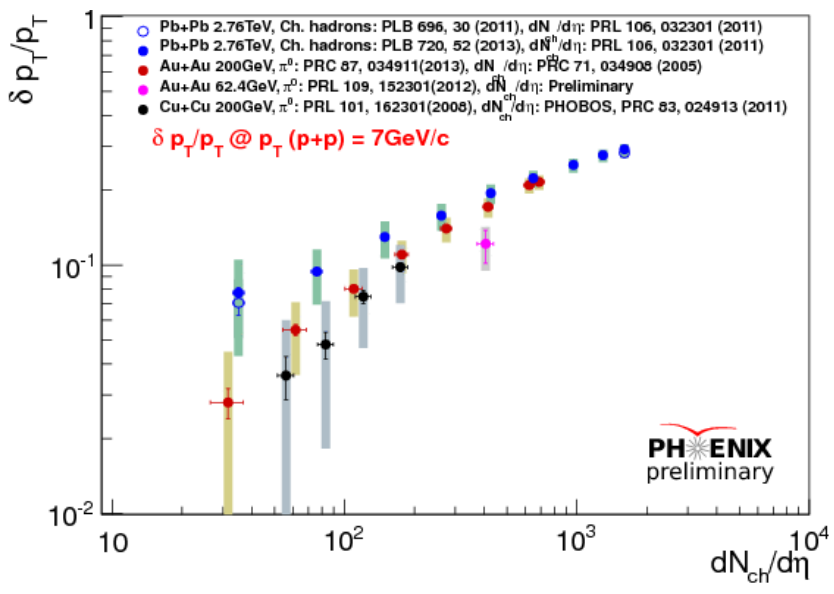

Fig.5. Fractional momentum loss as a function of particle multiplicity. 


\section{Production of hadrons at intermediate transverse momentum}

Fig. 6 shows the nuclear modification factors of $\pi^{0}, \eta, K^{ \pm}, K^{*}, K_{s}, \omega, \phi$ mesons and protons measured at intermediate transverse momentum in $\mathrm{Cu}+\mathrm{Cu}$ and $\mathrm{Au}+\mathrm{Au}$ collisions at $\sqrt{ }_{\mathrm{S}_{\mathrm{NN}}}=200 \mathrm{GeV}[2,10]$. In central heavy ion collisions production of baryons is enhanced while production of all mesons is significantly suppressed. There is a certain hierarchy in suppression of hadrons with no apparent mass or quark content dependence. Mesons containing strange quarks like $\mathrm{K}^{*}$ and $\phi$ show an intermediate suppression. The $\phi$ meson is closer to other mesons in most central collisions and is consistent with protons in peripheral interactions. At this $\phi$ is a meson that consists of two quarks and has a mass similar to that of a proton. In this sense comparison of results for protons and $\phi$ mesons is a subject of special interest since it can shed some light on the dominating hadron production mechanisms in this kinematic region which is very different from high $\mathrm{p}_{\mathrm{T}}$ region where production of all measured hadrons is similarly suppressed by about a factor of five.

Fig. 7 shows $p / \phi$ ratio measured as a function of transverse momentum. Plot on the left is for $\mathrm{Pb}+\mathrm{Pb}$ collisions at ${\sqrt{\mathrm{S}_{\mathrm{NN}}}}=2.76 \mathrm{TeV}$. Black points are for $\mathrm{p}+\mathrm{p}$, red and blue points are most central and peripheral $\mathrm{Pb}+\mathrm{Pb}$ collisions. We can see that ratio is pretty much the same in pp and peripheral $\mathrm{Pb}+\mathrm{Pb}$ collisions. In central $\mathrm{Pb}+\mathrm{Pb}$ collisions the $\mathrm{p} / \phi$ ratio is a flat function of

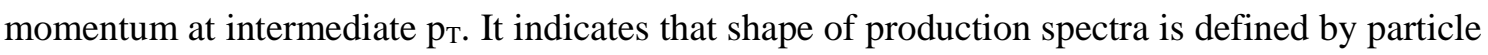
masses, not by hadron type or quark content that is consistent with hydrodynamics. If it is so then the difference in $\mathrm{R}_{\mathrm{AA}}$ values between protons and $\phi$ mesons is just driven by difference in $p+p$ references. Plot on the right shows $p / \phi$ ratio in $p+p$ and most central $A u+A u$ collisions at ${\sqrt{\mathrm{s}_{\mathrm{NN}}}}=200 \mathrm{GeV}$. Peripheral $\mathrm{Au}+\mathrm{Au}$ collisions are consistent with $\mathrm{p}+\mathrm{p}$ and are not shown for clarity. We see a similar evolution of $\mathrm{p} / \phi$ ratio from $\mathrm{p}+\mathrm{p}$ to central $\mathrm{Au}+\mathrm{Au}$ collisions at lower energy, although full flattening of the ratio is not achieved that makes interpretation more difficult.
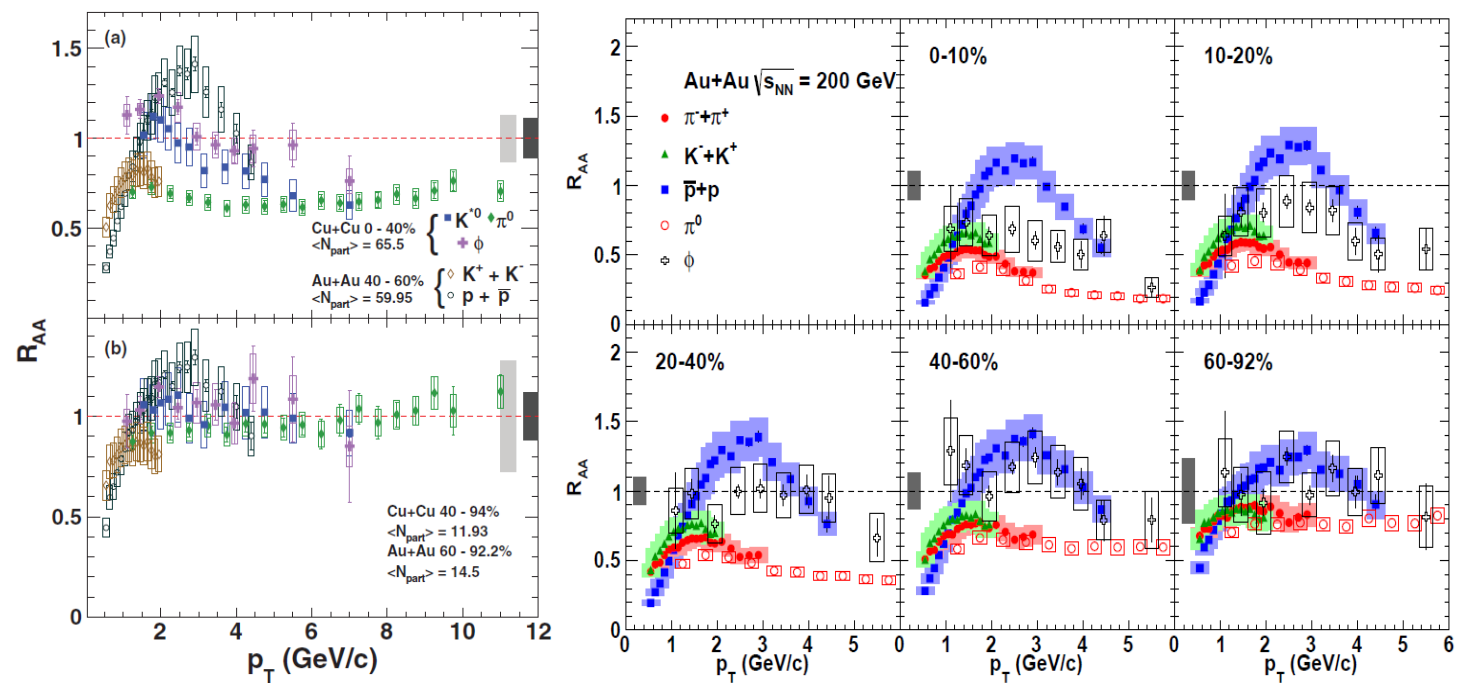

Fig.6. The nuclear modification factors of $\pi^{0}, \eta, K^{ \pm}, K^{*}, K_{s}, \omega, \phi$ mesons and protons measured at intermediate $\mathrm{p}_{\mathrm{T}}$ in $\mathrm{Cu}+\mathrm{Cu}$ and $\mathrm{Au}+\mathrm{Au}$ collisions at ${\sqrt{\mathrm{S}_{\mathrm{NN}}}}=200 \mathrm{GeV}$. 


\section{Direct photons}

By direct photons we mean all photons except for those coming from hadron decays. Such photons are produced at all stages of the collisions. The strongly interacting medium produced in heavy ion collisions is transparent for photons, they leave interaction region unaffected and therefore they serve as a good probe.
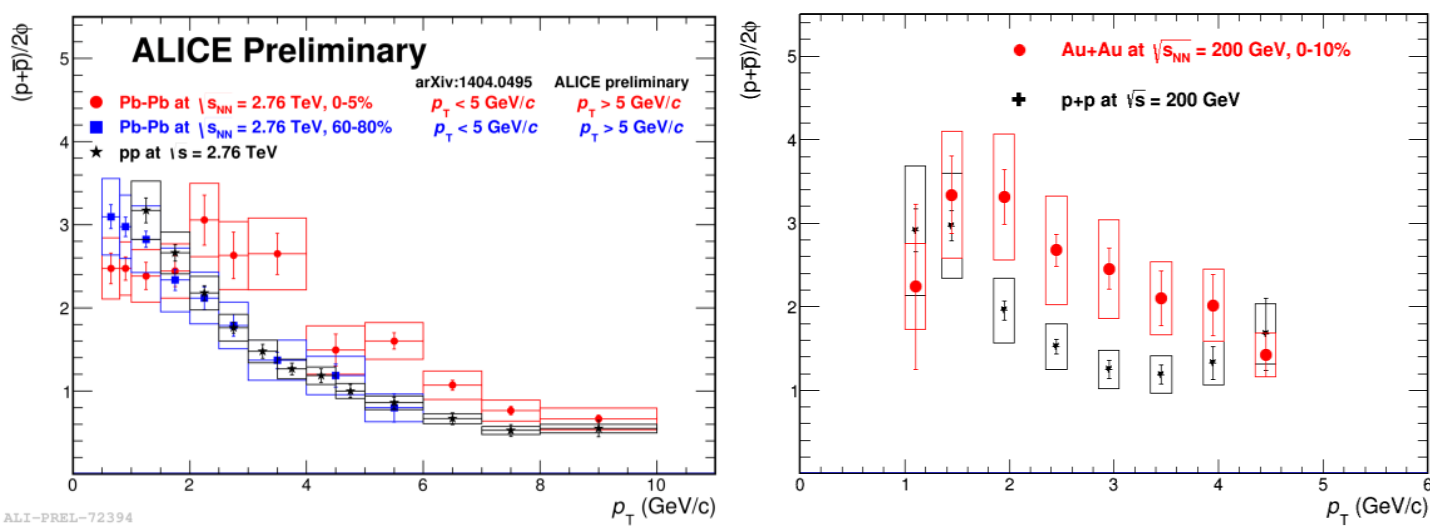

Fig.7. The $\mathrm{p} / \phi$ ratio measured as a function of transverse momentum in $\mathrm{Pb}+\mathrm{Pb}$ collisions at ${\sqrt{\mathrm{S}_{\mathrm{NN}}}}=2.76 \mathrm{TeV}$ (left) and in $\mathrm{Au}+\mathrm{Au}$ collisions at ${\sqrt{\mathrm{S}_{\mathrm{NN}}}}=200 \mathrm{GeV}$ (right)

In Fig. 8 you can see the nuclear modification factors measured for direct photons [11,12]. Plot on the top shows $R_{d A}$ of direct photons in minimum bias $d+A u$ collisions at $\sqrt{ }_{\mathrm{S}_{\mathrm{NN}}}=200 \mathrm{GeV}$. Plot on the bottom shows similar result for most central Au+Au collisions at the same energy. Within uncertainties $R_{d A}$ and $R_{A A}$ are consistent with unity in the whole range of measurements. Measured values of the factors can be quantitatively described by model calculations. Most of calculations in heavy ion collisions predict small suppression of direct photon production at high $\mathrm{p}_{\mathrm{T}}$ due to Isospin effect which originates from the different photon cross sections in $\mathrm{p}+\mathrm{p}, \mathrm{n}+\mathrm{n}$, and $\mathrm{p}+\mathrm{n}$ collisions. Modifications of nuclear structure functions and plasma effects do not change predictions much. In d+Au the theory calculations with different combinations of standard CNM effects predict similar results consistent with experimental data. In $R_{d A}$ there is a place for Cronin-like enhancement at intermediate $p_{T}$. If it is so it would support idea that Cronin is an initial state effect, because alternative models of recombination in the final state are not applicable for direct photons. Absence of suppression for high $\mathrm{p}_{\mathrm{T}}$ direct photons in central $\mathrm{Au}+\mathrm{Au}$ collisions was one of the most convincing arguments in favor of jet quenching as a main mechanism responsible for suppression of high $\mathrm{p}_{\mathrm{T}}$ hadrons.

Production of soft direct photons was measured in $\mathrm{p}+\mathrm{p}$ and $\mathrm{Au}+\mathrm{Au}$ collisions at ${\sqrt{\mathrm{S}_{\mathrm{NN}}}}=200 \mathrm{GeV}$ [13]. It was discovered that soft photon yields in $\mathrm{Au}+\mathrm{Au}$ collisions significantly exceed yields in $\mathrm{p}+\mathrm{p}$ scaled by the number of binary collisions $\mathrm{N}_{\text {coll }}$ at $\mathrm{p}_{\mathrm{T}}<4$ GeV/c. Recent measurements from PHENIX are shown in Fig. 9 [14]. They are in good agreement with previous results and provide wider $\mathrm{p}_{\mathrm{T}}$ range, more centrality selections and higher precision. Blue points in the figure are for soft direct photon yields in $\mathrm{Au}+\mathrm{Au}$ and greed curves are the Ncoll scaled $p+p$ results. In all centrality bins we observe excessive yield of photons. If we fit this excess yield to exponential function we will get slope parameters equal to 

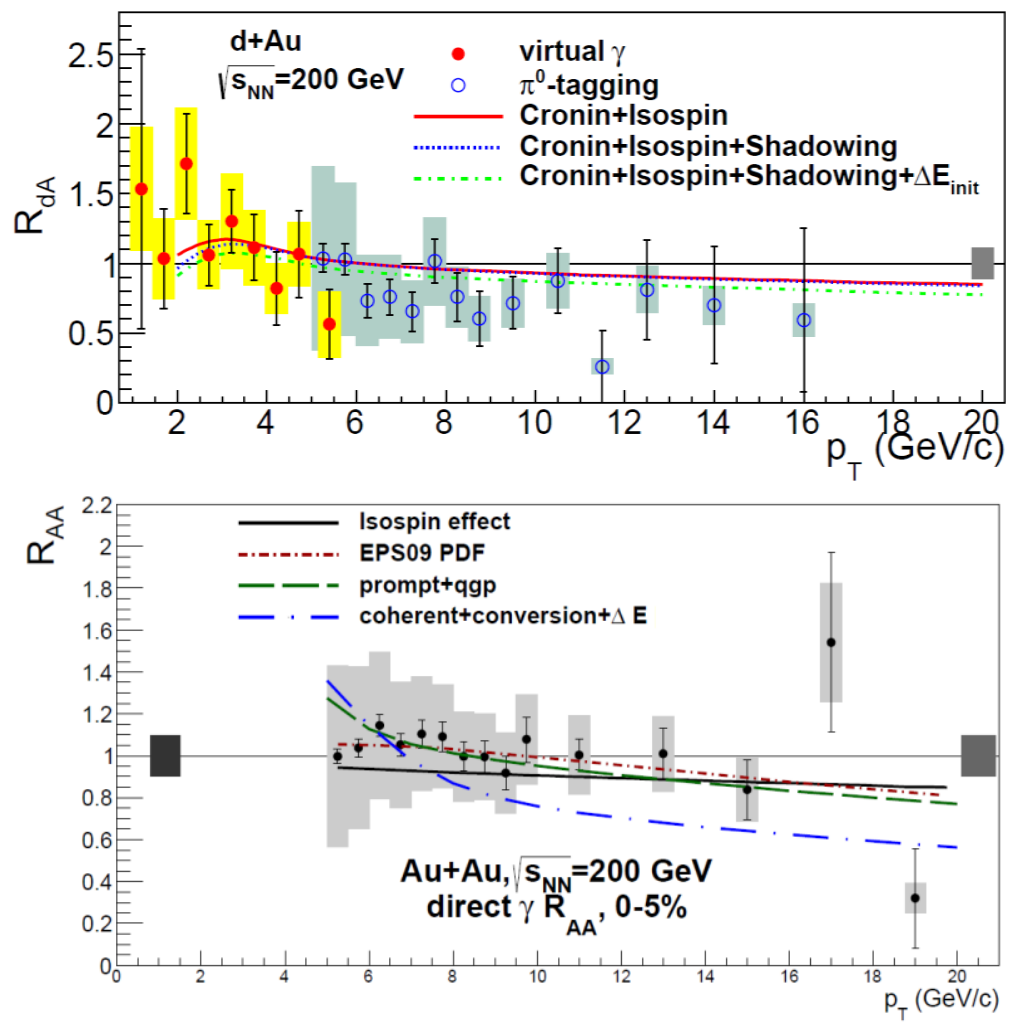

Fig.8. The nuclear modification factors measured for direct photons in $\mathrm{d}+\mathrm{Au}$ (top) and $\mathrm{Au}+\mathrm{Au}$ (bottom) collisions at ${\sqrt{\mathrm{S}_{\mathrm{NN}}}}=200 \mathrm{GeV}$.



Fig. 9. Direct photon pT spectra in $\mathrm{Au}+\mathrm{Au}$ collisions at ${\sqrt{\mathrm{S}_{\mathrm{NN}}}}=200 \mathrm{GeV}$.

Panels correspond to different centrality bins: 0-20\%, 20-40\%, 40-60 and 60-92\%. 
$\sim 240 \mathrm{MeV}$ with only very weak centrality dependence. Excess of photon yields scales with number of participants $\mathrm{N}_{\text {part }}$ as $\mathrm{N}_{\text {part }}{ }^{\alpha}$, where $\alpha=1.48 \pm 0.08$ (stat) \pm 0.04 (syst). Most of theoretical models need to suggest very early emission from the interacting system when temperature is still very high in order to describe large excessive yield of direct photons.

The PHENIX also has new measurements for direct photons anisotropy at low $\mathrm{p}_{\mathrm{T}}$. Top panel of Fig. 10 shows dependence of elliptic flow, $v_{2}$, on transverse momentum in $\mathrm{Au}+\mathrm{Au}$ collisions at ${\sqrt{\mathrm{S}_{\mathrm{NN}}}}=200 \mathrm{GeV}$ for different centrality bins. Panel on the bottom shows similar results for triangular flow, v $_{3}$. Flow was measured using two different methods, results are shown with green and red markers, and they agree very well in the overlap region. What is surprising is that values of $v_{2}$ and $v_{3}$ measured for soft direct photons are very similar to the values previously measured for light hadrons. Similar to hadrons the $v_{3}$ has only very weak dependence on collision centrality. Large measured values of $v_{2}$ and $v_{3}$ similar to that for light hadrons present a serious challenge for model calculations which should assume late emission when temperature is low but collective motion is large. So observations of the large photon yields and large flow are difficult to reconcile and there is still no satisfactory model description yet.
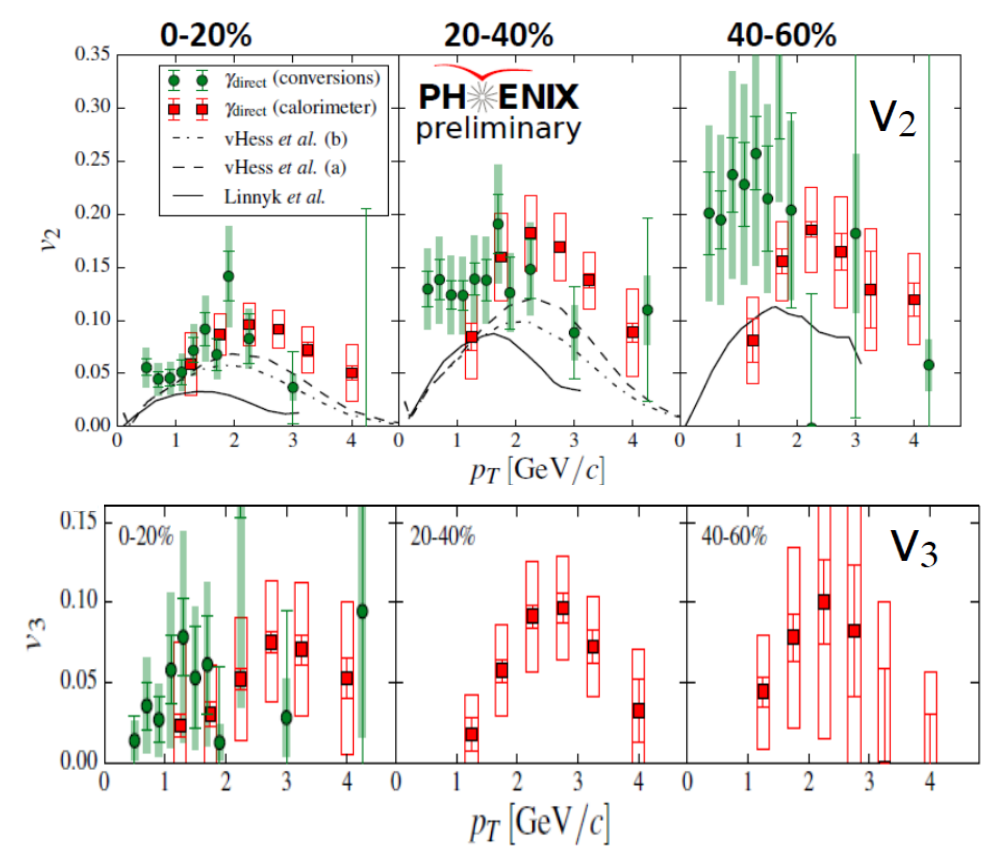

Fig. 10. Elliptic and triangular flow for soft direct photons in $\mathrm{Au}+\mathrm{Au}$ collisions at ${\sqrt{\mathrm{S}_{\mathrm{NN}}}}=200 \mathrm{GeV}$. 


\section{References}

[1] K. Adcox et al., Centrality Dependence of Charged Particle Multiplicity in Au-Au Collisions at $\sqrt{s_{N N}}=130 \mathrm{GeV}$, Phys. Rev. Lett. 86, 3500 .

[2] A. Adare et al., Measurement of $K_{S}$ and $K^{*}$ in $p+p, d+A u$, and $C u+C u$ collisions at ${\sqrt{s_{N N}}}=200 \mathrm{GeV}$, Phys.Rev. C90 (2014) 5, 054905.

[3] Rudolph C. Hwa et al., Scaling behavior at high $p(T)$ and the p/pi ratio, Phys.Rev. C67 (2003) 034902.

[4] A. Adare et al., Neutral pion production with respect to centrality and reaction plane in $A u+A u$ collisions at $\sqrt{s_{N N}}=200$ GeV, Phys.Rev. C87 (2013) 3, 034911.

[5] A. Adare et al., Suppression pattern of neutral pions at high transverse momentum in $\mathrm{Au}+\mathrm{Au}$ collisions at $\sqrt{ }_{s_{N}}=200 \mathrm{GeV}$ and constraints on medium transport coefficients, Phys.Rev.Lett. 101 (2008) 232301.

[6] A. Adare et al., Onset of piO Suppression Studied in $C u+C u$ Collisions at $\sqrt{ } s_{N N}=22.4,62.4$, and 200 GeV, Phys.Rev.Lett. 101 (2008) 162301.

[7] A. Adare et al., Evolution of $\pi 0$ suppression in $A u+A u$ collisions from $1 s_{N N}=39$ to $200 \mathrm{GeV}$, Phys.Rev.Lett. 109 (2012) 152301.

[8] K. Aamodt et al., Suppression of Charged Particle Production at Large Transverse Momentum in Central Pb--Pb Collisions at ${\sqrt{s_{N N}}}=2.76 \mathrm{TeV}$, Phys.Lett. B696 (2011) 30-39.

[9] K. Adcox et al., Formation of dense partonic matter in relativistic nucleus-nucleus collisions at RHIC: Experimental evaluation by the PHENIX collaboration, Nucl.Phys. A757 (2005) 184-283.

[10] A. Adare et al., Spectra and ratios of identified particles in $A u+A u$ and $d+A u$ collisions at $V_{s_{N N}}$ =200 GeV, Phys.Rev. C88 (2013) 2, 024906.

[11] A. Adare et al., Direct photon production in $d+A$ u collisions at $\sqrt{s_{N N}}=200 \mathrm{GeV}$, Phys.Rev. C87 (2013) 054907.

[12] S. Afanasiev et al, easurement of Direct Photons in Au+Au Collisions at $\widehat{ }_{s_{N N}}=200 \mathrm{GeV}$, Phys.Rev.Lett. 109 (2012) 152302.

[13] A. Adare et al., Detailed measurement of the $\mathrm{e}+\mathrm{e}-$ pair continuum in $\mathrm{p}+\mathrm{p}$ and $\mathrm{Au}+\mathrm{Au}$ collisions at $\sqrt{ }_{s_{N N}}=200 \mathrm{GeV}$ and implications for direct photon production, Phys.Rev. C81 (2010) 034911.

[14] A. Adare et al., Centrality dependence of low-momentum direct-photon production in $A u+A u$ collisions at $\sqrt{s_{N N}}=200 \mathrm{GeV}$, [arXiv:1405.3940]. 